http://jmscr.igmpublication.org/home/

ISSN (e)-2347-176x ISSN (p) 2455-0450

crossref DOI: https://dx.doi.org/10.18535/jmscr/v9i8.15

Journal Of Medical Science And Clinical Research

IGM Publication

An Official Publication of IGM Publication

\title{
Cytomorphological Study Emphasizing Early Diagnosis of Mesothelioma in a Tertiary Care Centre in Southern Rajasthan
}

\author{
Author
}

\section{Dr Rini Bishnoi}

Senior Demonstrator, Pathology Department, Government Medical College, Pali, India

\begin{abstract}
Background: Malignant Mesothelioma (MM) is a rare and aggressive tumour of the pleura and peritoneum associated with short survival time and needs timely diagnosis which varies with different histological subtypes.

Aim: To evaluate the incidence and clinicomorphological features of malignant mesothelioma with emphasis on early diagnosis by cytology.

Materials and Methods: A total of 78 cases of malignant mesotheliomas of different sites encountered over a 2-year period were retrospectively analysed with regard to their cytological findings and subsequent histopathology subtyping and follow up with patient survival.

Results: Environmental asbestos exposure was found in $75.00 \%$ of patients. The pleura was the most common site of involvement (76 cases)with one case of pericardial and peritoneal mesothelioma. Epithelioid mesothelioma was the most common histological type (62 cases, 79.4\%) followed by sarcomatoid, desmoplastic and biphasic (4 cases each, 6.4\%)and deciduoid (2 cases, 3.2\%). All the cases diagnosed on FNA/Effusion cytology were confirmed by histopathology and subtyping was done. Mean patient survival time was 9 months.

Conclusion: Malignant mesothelioma related to asbestos exposure is seen rampantly in active mining areas of Southern Rajasthan and is associated with poor survival rates, which emphasizes the need for early diagnosis and histological subtyping for better patient outcomes.

Keywords: Histological type;cytology; mesothelioma.
\end{abstract}

\section{Introduction}

Mesotheliomas are aggressive malignant neoplasms arising from theserosal lining of the body cavities. MM is caused mainly by the prolonged inhalation of asbestos fibres in patients working in mining industry. Latency from first exposure varies from 15 to 50 years, there is no cure and median survival is $10-12$ months. ${ }^{1,2}$ Asbestos utilisation continues to increase in many developing countries. ${ }^{3}$
The most common sites of involvement are the pleura $(60 \%-70 \%)$ followed by peritoneum $(30 \%-35 \%)$, and pericardium $(0.7 \%)$. Diagnosis requires a multimodal approach that includes clinical findings, imaging studies, effusion cytology, FNA, histopathology and immunohistochemistry (IHC).

Patients most commonly present with dyspnoea and chest pain. Dyspnoea occurs due to restriction caused by thickened pleuraoraccumulation of pleural fluid. Pain is often expansive and obtuse 
on the lateral wall of the chest, generally chronic, persistent, and nonpleuretic. ${ }^{4}$

After appropriate clinical history and imaging, diagnosis of malignant mesothelioma (MM) can in the majority of cases be based on the evaluation of the fine needle aspiration of pleural mass or effusion cytology as the first non-invasive investigation for an unexplained pleural or peritoneal effusion in most cases. This allows early diagnosis of a malignant pleural/peritoneal effusion in many cases.

Malignant mesothelioma has distinctive histological subtypes: epithelioid, sarcomatoid and biphasic (composed of both epithelioid and sarcomatoid components). The diagnosis of MM using According to the Consensus Statement of the International Mesothelioma Interest Group, 'The diagnosis of MM must always be based on the results obtained from an adequate biopsy in the context of appropriate clinical, radiologic and surgical findings, 5

Epithelioid MM (the most common form) more readily sheds cells into the pleural or peritoneal space and these can be identified on cytological examination. By contrast, sarcomatoid MM cells usually do not shed into the pleural or peritoneal space, leading to a poor cytological diagnostic yield for the sarcomatoid subtype and the possibility of misclassification of biphasic MM as epithelioid MM using the cytology-only approach. The pathological subtype of MM has a strong influence on survival with the epithelioid type associated with a longer survival as compared with biphasic and sarcomatoid $\mathrm{MM}^{6}$

A cytological diagnosis of MM, however, has practical advantages including that it is minimally invasive, easily performed and inexpensive.

\section{Materials and Methods}

In all, 78 cases of mesotheliomas diagnosed at the Department of Pathology at Geetanjali medical college, Udaipur over a period of 2 years from January 2016 to January 2018 were analysed retrospectively. The demographic data, clinical features, and imaging findings were retrieved from medical records. Diagnosis of pleural mesothelioma made on FNA or effusion cytology was confirmed histologically on pleural biopsies which included both thoraco-scopic and core needle.

Case of pericardial mesothelioma was diagnosed on biopsy specimen. Abdominal mesothelioma case was diagnosed on biopsy specimens submitted following exploratory laparotomy. All the specimens were received and fixed in neutral buffered formalin, routinely processed for paraffin embedding. The haematoxylin and eosin $(\mathrm{H}$ and E)-stained sections along with IHC slides were reviewed in all the cases. The panel of IHC markers was performed wherever necessary.

\section{Results}

Age, gender, clinical features, site and histological subtype in all 78 cases of MMs are listed in Table (1-5) The age of the patients ranged from 22 to 88 years with a peak incidence in sixth to eighth decade. There was male preponderance with a M:F ratio of 1.6:1. The most common site was pleura (16 cases) followed by peritoneum and pericardium 1 case each. $75 \%$ of cases had occupational/environmental exposure to asbestos. The clinical presentation varied depending on the site of involvement. Patients with pleural mesotheliomas primarily presented with dry cough, shortness of breath, and chest pain. There was pleural effusion with multiple nodules or diffuse pleural thickening. Patient of pericardial mesotheliomas presented with chronic constrictive pericarditis, cardiac tamponade followed by heart failure. The patient of peritoneal mesotheliomas presented with abdominal pain and ascites. The age of the patients ranged between 22 years to 85 years in the present study, with maximum number of 22 cases seen in age range from 61-70 years, followed by 20 cases in 51-60 years. (Table 1) 
Table 1: Age distribution of cases in the present study

\begin{tabular}{|l|c|c|}
\hline Age group (years) & Number of cases & \% of cases \\
\hline $20-30$ & 4 & 5.1 \\
\hline $31-40$ & 6 & 7.6 \\
\hline $41-50$ & 5 & 6.4 \\
\hline $51-60$ & 20 & 25.6 \\
\hline $61-70$ & 22 & 28.20 \\
\hline $71-80$ & 16 & 20.5 \\
\hline $81-90$ & 5 & 6.4 \\
\hline Grand Total & 28 & $100.00 \%$ \\
\hline
\end{tabular}

Sex distribution (Table 2): In the present study there was slight male preponderance $(61.5 \%)$.

Table 2: Sex distribution of cases in the present study

\begin{tabular}{|c|c|c|}
\hline Gender & Number of cases & \% of cases \\
\hline Males & 48 & $61.5 \%$ \\
\hline Females & 30 & $38.4 \%$ \\
\hline Total & 78 & 100 \\
\hline
\end{tabular}

Clinical features (Table 3)-Majority of patients $(89.7 \%$ ) presented with complaints of dyspnoea and chest pain $(66.6 \%)$ to OPD whereas $52.5 \%$ complained of dry cough without expectoration. $15 \%$ patients presented with a fever and $48.3 \%$ with associated loss of appetite. 1 case of abdominal mesothelioma presented with abdominal pain.

$40 \%$ presented with history of smoking for $20-30$ years.

Table 3: Clinical features of the cases in the present study

\begin{tabular}{|c|c|c|}
\hline Clinical features & Number of cases & \% of cases \\
\hline Dyspnoea & 70 & 89.7 \\
\hline Chest pain & 52 & $66.6 \%$ \\
\hline Dry cough & 48 & $57.6 \%$ \\
\hline Fever & 12 & $15.3 \%$ \\
\hline Loss of appetite & 38 & $48.71 \%$ \\
\hline Abdominal pain & 1 & $1.28 \%$ \\
\hline
\end{tabular}

Site of the lesion (Table 5) -Majority of cases were from pleural region with one case each from pericardium and peritoneum.

Table 5. Distribution of the cases according to site Cytological findings

\begin{tabular}{|c|c|c|}
\hline Site & Number of cases & $\%$ of cases \\
\hline Pleura & 76 & $97.4 \%$ \\
\hline Pericardium & 1 & $1.28 \%$ \\
\hline Peritoneum & 1 & 1.28 \\
\hline Total & 78 & $100 \%$ \\
\hline
\end{tabular}

Air-dried and alcohol-fixed smears were prepared. The MGG stained aspirate smears were hypercellular with cells arranged in loose clusters and singly dispersed in a background of granular hyaline like material [Figure 1]. The cells had a low nuclear to cytoplasmic ratio, moderate to abundant dense cytoplasm, and distinct cell borders. Some cell clusters showed intercellular windows [Figure 2]. Nuclei were centrally to eccentrically placed, with moderate anisonucleosis and frequent binucleation. Cytoplasmic vacuoles were readily appreciated, most commonly as multiple small vacuoles located near the nucleus.

Some smear showed cells arranged in tubulopapillary as well as syncytial pattern and singly dispersed cells. These cells had moderate pleomorphism with nuclear atypia.

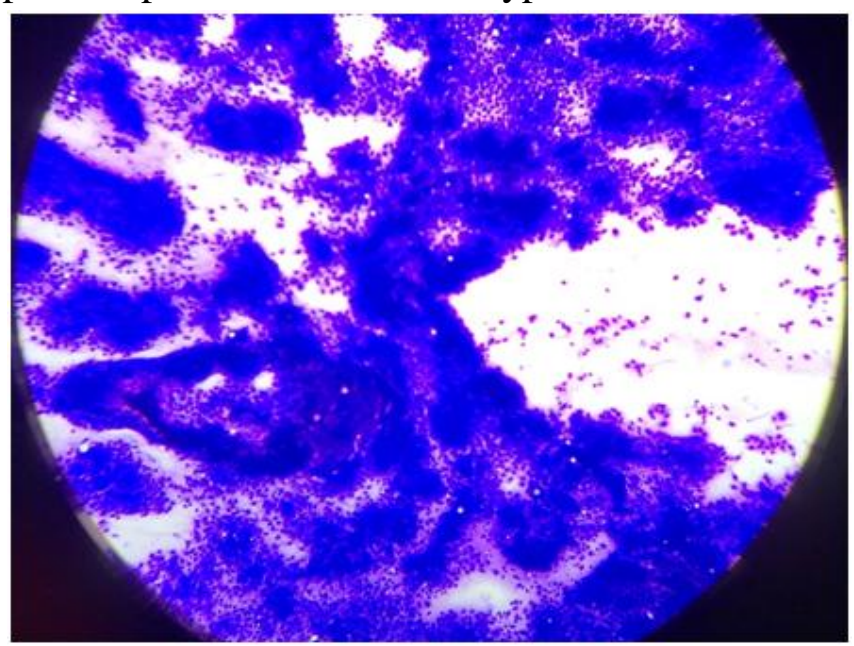

Figure 1. Hypercellular smear showing large and small tissue fragments

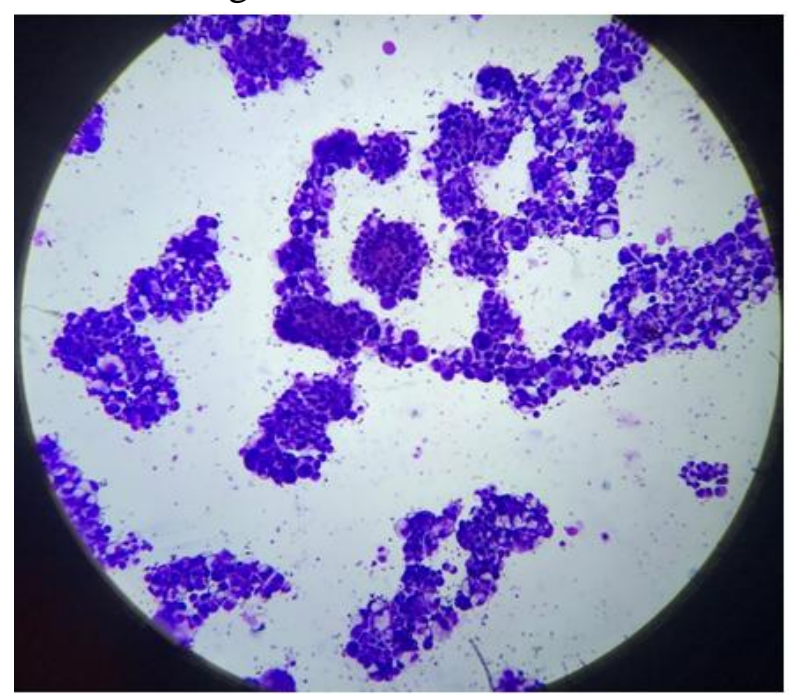

Figure 2 Cytology smear showing prominent cell separation-pavement like pattern 


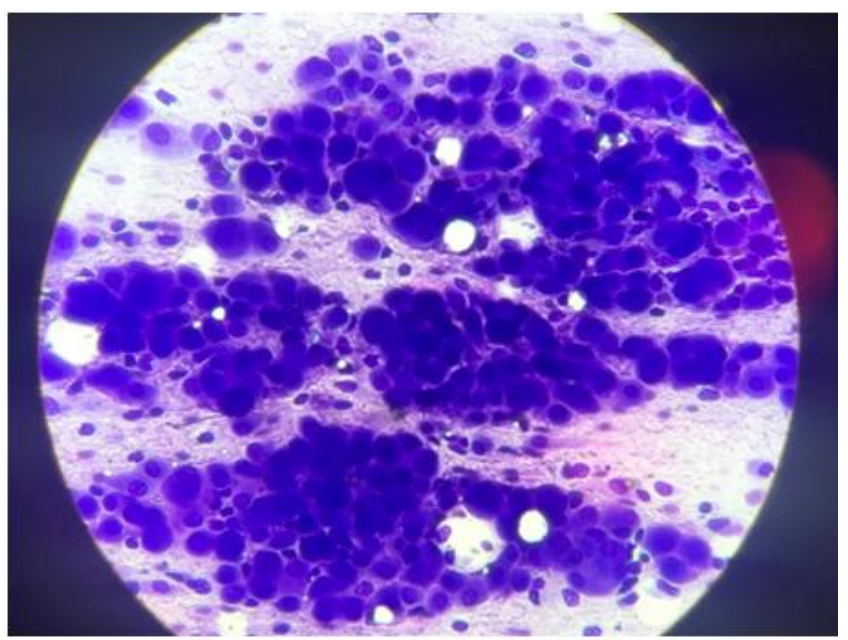

Figure 3 Cytomorphology of the epithelioid mesothelioma cells in effusions. The malignant mesothelioma cells often show macronucleoli. and there are prominent degree of cell-within-cell arrangements and the tumor cells are sometimes multinucleated

Epithelioid mesothelioma was the commonest histological type (62 cases, 79.4\%) followed by sarcomatoid (4 cases, 6.4\%), deciduoid (2 cases, $2.3 \%)$. and 4 cases each of biphasic and poorly differentiated mesothelioma. While epithelioid mesotheliomas involved all three sites, the sarcomatoid, and biphasic subtypes were confined only to the pleura. Single case of pericardial mesothelioma were of epithelioid subtype and both deciduoid mesotheliomas encountered involved the peritoneum.

Histopathologic Diagnosis_(Table 8): Among 78 cases majority of cases were Epithelioid type 62 cases $(79.4 \%), 6(7.6 \%)$ cases were of Sarcomatous type, $4(6.4 \%)$ cases each of Biphasic type and Poorly differentiated type.

Table 8: Histopathological subtypes of mesothelioma

\begin{tabular}{|c|c|c|c|c|}
\hline \multicolumn{2}{|c|}{ S.No } & Histopathological type & No. & $\%$ \\
\hline 1 & & & & \\
\hline \multirow{5}{*}{} & a & Epithelial & 62 & $79.4 \%$ \\
\cline { 2 - 5 } & b & Sarcomatous & 4 & $6.4 \%$ \\
\hline & c & Decidouid & 2 & $3.2 \%$ \\
\hline & c & Biphasic & 4 & $6.4 \%$ \\
\cline { 2 - 5 } & e & Poorly differentiated & 4 & $6.4 \%$ \\
\hline
\end{tabular}

On histology, epithelioid mesotheliomas showed various architectural patterns ranging from tubulo- papillarybeing the commonest to acinar and diffuse pattern. (Figure 3,4). The sarcomatoid mesotheliomawas comprised of sheets and fascicles of pleomorphic spindle cells with mitosis and necrosis.The deciduoid mesotheliomas comprised sheets of large, round to polygonal cells with abundant glassy eosinophilic cytoplasm and round vesicular nuclei with prominent nucleoli. The biphasic subtype had a combination of epithelioid and sarcomatoid subtypes.
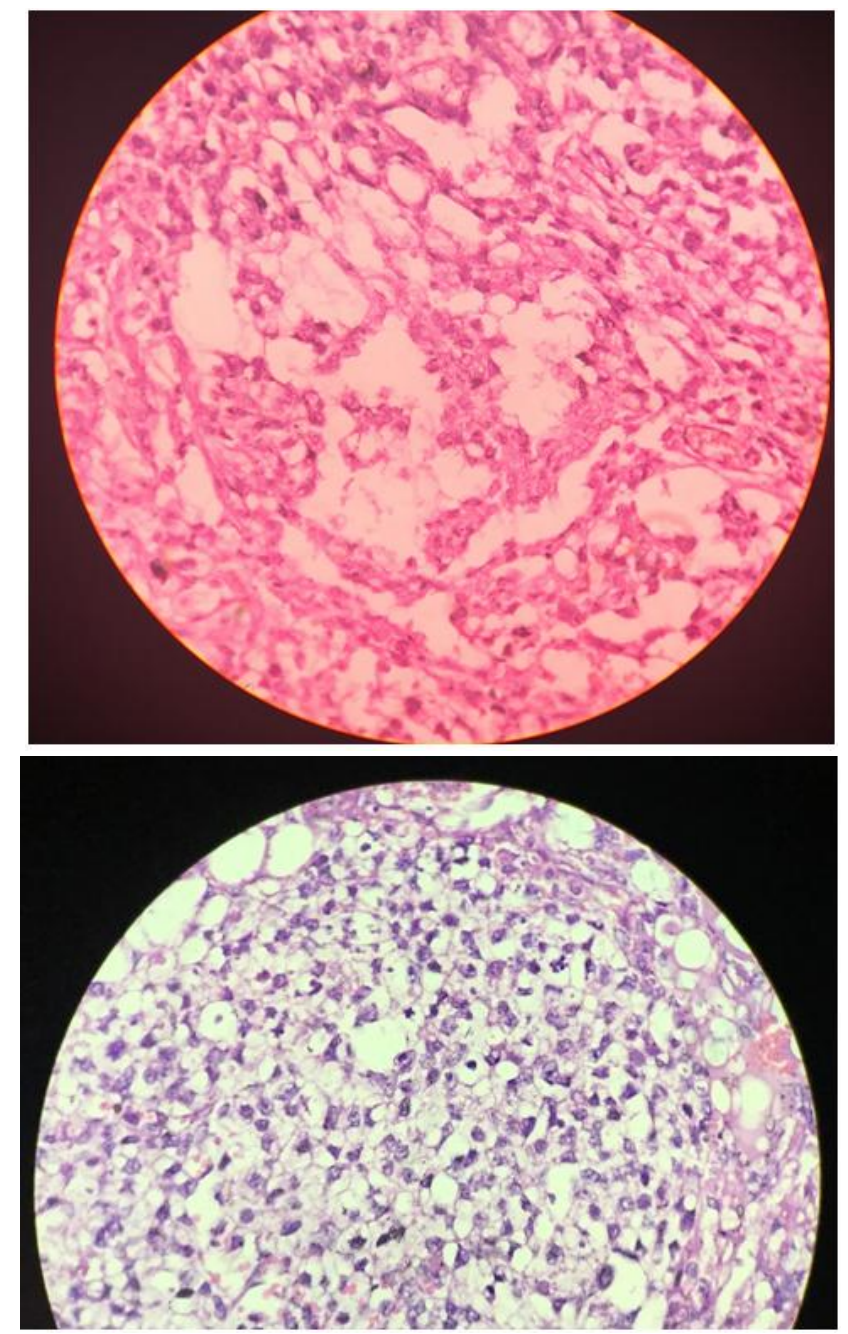

Figure 4 (a,b) Epithelioid type MM showing large polygonal sheets and tubule-papillary growth pattern

As epithelioid mesotheliomas display a variety of histological pattern, its distinction from adenocarcinomas involving the serosal cavities is difficult on light microscopy. There are no established guidelines for the use of specific antibody panels. A combination of two "positive" 
mesothelial and two "negative" carcinoma markers has been advocated by the International Mesothelioma Panel. The choice of IHC markers to be included in the panel varies depending on the location, histologic type, and the differential diagnosis considered in each case. ${ }^{[16]}$ The primary differential diagnosis for pleural epithelioid mesothelioma is metastatic lung adenocarcinoma. The use of epithelial markers in combination with the mesothelial markers is thus required for a definite diagnosis. In our experience, a panel of CEA, TTF1 and napsin A in combination with mesothelial markers (calretinin, CK5/6, D2-40, and WT-1) was useful to rule out pulmonary adenocarcinoma. (Figure 5)
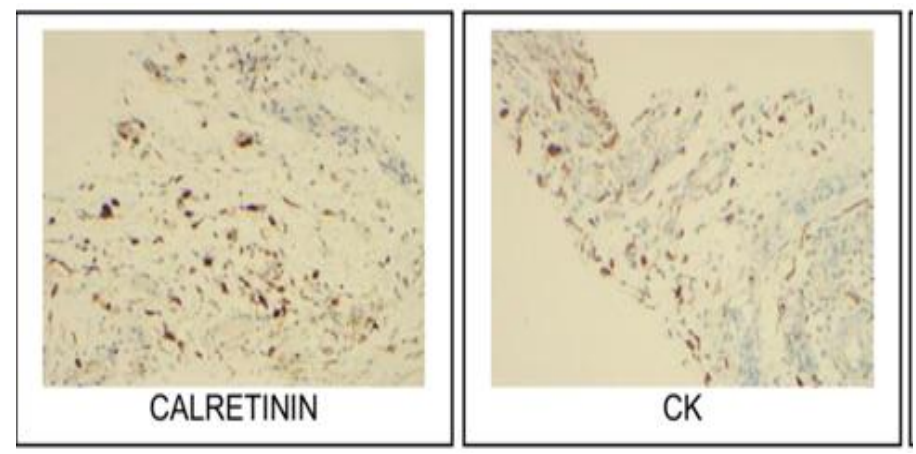

Figure 5 - Calretinin - Immunoreactive, Score 1+ in lesionalcells

\section{Discussion}

MMs are rare, histologically heterogeneous neoplasm predominantly seen in males with a peak age incidence in the fifth to sixth decade. ${ }^{7}$ The most common aetiology in cases of pleural and peritoneal mesotheliomas is linked to asbestos exposure with a long period of latency ranging from 30 to 40 years.

It has an insidious onset with clinical presentation ranging from pleuritic chest pain, dyspnoea, dry cough, fatigue, and weight loss. ${ }^{8}$

The peritoneal mesothelioma presents with vague symptoms such as abdominal pain, distention, anorexia, and weight loss and thus leads to advanced disease at presentation.

Radiological findings of CECT chest reveal irregular diffuse pleural thickening with or without massive pleural effusion displacing the mediastinal dome. Kawashima et alin their series of 50 cases of MM found pleural thickening in 92\% cases and thickening of the pleural surfaces of the interlobar fissures in $86 \%$ cases $^{9}$. They also noticed pleural effusion (74\%), calcifications (20\%), and chest wall invasion (18\%). Positron emission tomography is effective in differentiating MM from a reactive process and is also useful for staging. ${ }^{10}$

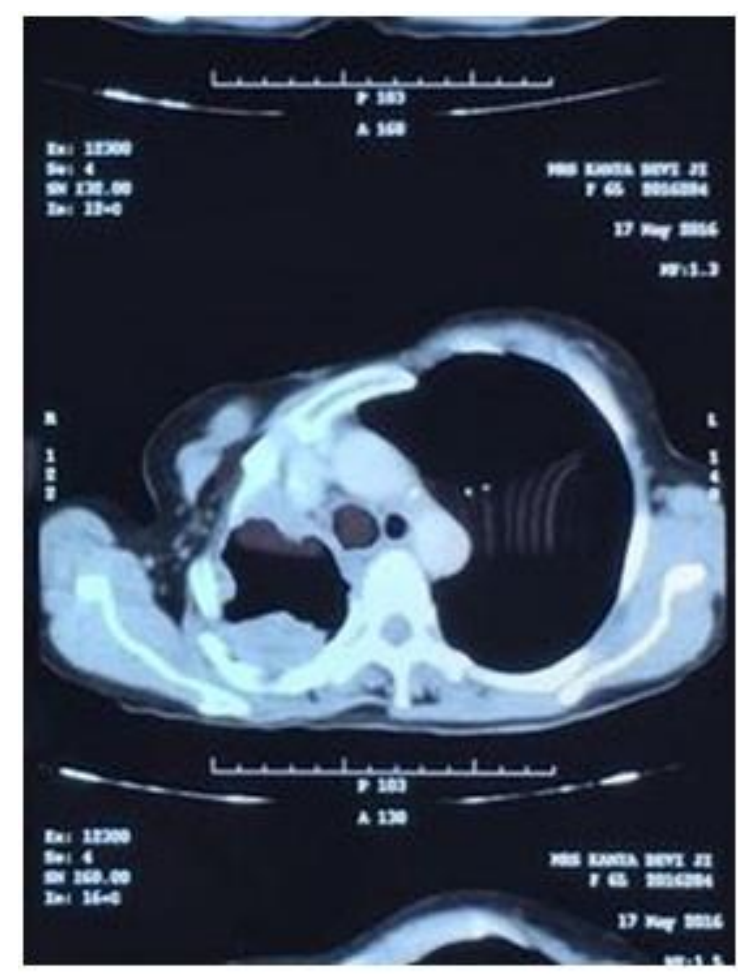

Figure 6 - CECT chest showing diffuse irregular pleural thickening with fibrosis and volume loss of right lung and displaced mediastinum.

Tumours diffusely infiltrating the pleura, pericardium or peritoneum can mimic MM clinically and radiologically. Histology is the gold standard for diagnosis along with IHC markers. In these cases an adequate biopsy should be interpreted in the background of appropriate clinical, radiologic, and surgical findings. There is significant morphological overlap between a reactive mesothelial proliferation and MM. Both can have papillary configuration with nuclear atypia and entrapment of cells within a fibrous stroma mimicking a neoplasm. In such cases, definite diagnosis is made though the presence of stromal or fat invasion. All the cases in our study 
were large expansile masses with definite evidence of stromal and or fat invasion excluding the possibility of reactive mesothelial proliferation.

Current reporting guidelines cite concerns with regard to using cytology as a means of diagnosing MM and it is widely accepted that this method is not useful for the diagnosis of sarcomatoid type tumours. There is sighted evidence that a cytological diagnosis of MM supported with supplementary techniques such as immunohistochemistry is as reliable as histopathological diagnosis ${ }^{11}$. Erroneous diagnosis of biphasic MM as epithelioid can be made, because the sarcomatoid component cells are not shed into the pleural space, and, it affects survival outcome when compared with biphasic or sarcomatoid MM.

In cytologicaly diagnosed cases of epithelioid type $\mathrm{MM}$, the morbidity of the patients specifically the older age group (>70 years),is significantly decreased since a definitivediagnosis negates the need for more invasive diagnostic procedures and eliminate the necessity for a biopsy, which has a higher potential for morbidity and a documented increased risk of tumor seeding. Definitive treatment can also be initiated avoiding delay. However, when effusion cytology is inconclusive for the diagnosis of MM, tissue core biopsy should be performed as previously recommended. The two techniques are complementary.

There are relatively few studies sensitivity of mesothelioma diagnosis by effusion cytology. In the past, sensitivity of around 30\% are often quoted(Renshaw et al, 1997a) ${ }^{12}$, however, labs with expertise in areas of high incidence can achieve sensitivities of over $70 \%$ (Segal et al, $2013)^{13}$, and other more recent studies document sensitivities of 50-60\% (Rakha et al, 2010; Pinelli et al, 2012) ${ }^{14}$. The sensitivity of mesothelioma diagnosis by effusion/FNA cytology in our study $\sim 60-70 \%$ which was comparable to other studies. The recently added new immunohistochemical stains (BAP1) and FISH studies (CDKN2A/p16) will contribute in increasing the sensitivity of mesothelioma diagnosis in effusion samples in all laboratories (Walts et al, 2016) $1^{15}$.

\section{Conclusion}

MM related to asbestos exposure is found rampantly in active mining areas in Rajasthan. Patients present with the typical clinical features of dyspnoea, weight loss, and chest pain. Patients have poor survival time due to aggressive nature of MM. The results of this study demonstrate that cases with a diagnosis of MM using cytology specifically epithelioid type have no significant difference in overall survival as compared with cases with histologically diagnosed epithelioid MM.

MM has a poor prognosis no matter what treatment regimen is offered. Earlier studies determined average survival time as 6-12 months. Mean survival time was found to be 9.3 months in our study.

Effusion and FNA cytology in suspected MM cases is minimally invasive and inexpensive, which are further important considerations the population having prolonged asbestos exposure, given the limited life expectancy.

Therefore, cytological analysis offers advantage for patients and healthcare resources. Clinicians can reliably inform patients of the diagnosis and prognosis with the knowledge that there is no clinically meaningful difference between overall survival with either diagnostic technique.

\section{References}

1. Olsen NJ, Franklin PJ, Reid A, de Klerk NH, Threlfall TJ, Shilkin K, Musk B (2011) Increasing incidence of malignant mesothelioma after exposure to asbestos during home maintenance and renovation. Med J Aust 195(5): 271-274

2. Brims FJ, Maskell NA (2013) Prognostic factors for malignant pleural mesothelioma. Curr Respir Care Rep 2(2): 100-108. 
3. Kazan-Allen L (2005) Asbestos and mesothelioma: worldwide trends. Lung Cancer 49(Suppl 1): S3-S8.

4. Ahmed I, Koulaouzidis A, Iqbal J, Tan WC. Malignant peritoneal mesothelioma as a rare cause of ascites: a case report. J Med Case Reports. 2008;2:121.

5. Husain AN, Colby TV, Ordóñez NG, Krausz T, Borczuk A, Cagle PT, et al. Guidelines for pathologic diagnosis of malignant mesothelioma: A consensus statement from the International Mesothelioma Interest Group. Arch Pathol Lab Med 2009;133:1317-31.

6. Musk AW, Olsen N, Alfonso H, Reid A, Mina R, Franklin P, Sleith J, Hammond N, Threlfall T, Shilkin KB, de Klerk N (2011) Predicting survival in malignant mesothelioma. Eur Respir J 38(6): 14201424.

7. Marinaccio A, Binazzi A, Marzio DD, Scarselli A, Verardo M, Mirabelli D, et al. Pleural malignant mesothelioma epidemic: Incidence, modalities of asbestos exposure and occupations involved from the Italian National Register. Int J Cancer 2012;130:2146-54.

8. Rusch VW. Clinical features and current treatment of diffuse malignant pleural mesothelioma. Lung Cancer 1995;12(Suppl 2):S127-46.

9. Kawashima A, Libshitz HI. Malignant pleural mesothelioma: CT manifestations in 50 cases. Am J Roentgenol 1990;155:965-9. Back to cited text no. 14

10. Benard F, Sterman D, Smith RJ, Kaiser LR, Albelda SM, Alavi A. Prognostic value of FDG PET imaging in malignant pleural mesothelioma. J Nucl Med 1999;40:1241-5.
11. Rakha EA, Patil S, Abdulla K, Abdulkader M, Chaudry Z, Soomro IN (2010) The sensitivity of cytologic evaluation of pleural fluid in the diagnosis of malignant mesothelioma. Diagn Cytopathol 38(12): 874-879.

12. Renshaw AA, Dean BR, Antman KH, Sugarbaker DJ, Cibas ES (1997a) The role of cytologic evaluation of pleural fluid in the diagnosis of malignant mesothelioma. Chest 111(1): 106-109.

13. Segal A, Sterrett GF, Frost FA, Shilkin $\mathrm{KB}$, Olsen NJ, Musk AW, Nowak AK, Robinson BW, Creaney J (2013) A diagnosis of malignant pleural mesothelioma can be made by effusion cytology: results of a 20 year audit. Pathology 45(1): 44-48.

14. Pinelli V, Laroumagne S, Sakr L, Marchetti GP, Tassi GF, Astoul P (2012) Pleural fluid cytological yield and visceral pleural invasion in patients with epithelioid malignant pleural mesothelioma. J Thorac Oncol 7(3): 59559.

15. Walts AE, Hiroshima K, McGregor SM, Wu D, Husain AN, Marchevsky AM (2016) BAP1 immunostain and CDKN2A (p16) FISH analysis: clinical applicability for the diagnosis of malignant mesothelioma in effusions. Diagn Cytopathol 44(7): 599-606. 